OPEN ACCESS

Edited by:

Ming Li,

Zhejiang University, China

Reviewed by:

Mohammad Hossein Heydari, Shiraz University of Technology, Iran Yong Ren,

Anhui Normal University, China Yaozhong $\mathrm{Hu}$,

University of Alberta, Canada

*Correspondence: Xichao Sun sunxichao626@126.com

Specialty section:

This article was submitted to Interdisciplinary Physics, a section of the journal

Frontiers in Physics

Received: 25 September 2021 Accepted: 14 October 2021 Published: 22 November 2021

Citation:

Gao L and Sun X (2021) Almost

Periodic Solutions to Impulsive Stochastic Delay Differential Equations Driven by Fractional Brownian Motion With $\frac{1}{2}<H<1$.

Front. Phys. 9:783125.

doi: 10.3389/fphy.2021.783125

\section{Almost Periodic Solutions to Impulsive Stochastic Delay Differential Equations Driven by Fractional Brownian Motion With $\frac{1}{2}<\mathbf{H}<1$}

\author{
Lili Gao and Xichao Sun* \\ Department of Mathematics and Physics, Bengbu University, Bengbu, China
}

In this article, we study the existence and uniqueness of square-mean piecewise almost periodic solutions to a class of impulsive stochastic functional differential equations driven by fractional Brownian motion. Moreover, the stability of the mild solution is obtained. To illustrate the results obtained in the paper, an impulsive stochastic functional differential equation driven by fractional Brownian motion is considered.

Keywords: fractional Brownian motion, square-mean piecewise almost periodic solution, impulsive systems, stochastic functional differential equation, stability

\section{INTRODUCTION}

Impulsive systems arise naturally in a wide variety of evolutionary processes in which states are changed abruptly at certain moments of time. Impulsive stochastic modeling has come to play an important role in many branches of science where more and more people have encountered impulsive stochastic differential equations. For example, a stochastic model for drug distribution in a biological system was described by Tsokos and Padgett [1] as a closed system with a simplified heart, one organ, or capillary bed, and recirculation of blood with a constant rate of flow, where the heart is considered as a mixing chamber of constant volume. Recently, there has been a significant development in impulsive stochastic differential equations (ISDEs). The existence and stability of ISDEs were investigated in [2-11] and the references therein.

On the other hand, in recent years, there has been considerable interest in studying fractional Brownian motions (fBms) due to their compact properties and applications in various scientific areas, including telecommunications [12, 13], turbulence [14], image processing [15], and finance [16]. Stochastic differential equations (SDEs) driven by fBms attract the interest of researchers [2, 3 , 17-21]. Taking the time delay into account, the theory of stochastic differential equations has been generalized to stochastic functional differential equations; it makes the dynamics more complex and the system may lose stability and show almost periodicity. Arthi et al. [2] considered the existence and exponential stability for neutral stochastic integrodifferential equations with impulses driven by fractional Brownian motion ( $\mathrm{fBm}$ ), and Caraballo [3] studied the existence of mild solutions to stochastic delay evolution equations with $\mathrm{fBm}$ and impulses.

In this paper, we are concerned with the existence and stability of almost periodic mild solutions to the following impulsive stochastic functional differential system driven by $\mathrm{fBm}$ with Hurst index $H \in(1 / 2,1)$ :

$$
\left\{\begin{array}{l}
d x(t)=\left[A x(t)+b\left(t, x_{t}\right)\right] d t+\sigma(t) d B^{H}(t), \quad t \neq \pm t_{i}, \quad i \in \mathbb{Z}, \\
\triangle x\left(t_{i}\right)=x\left(t_{i}^{+}\right)-x\left(t_{i}^{-}\right)=I_{i}\left(x\left(t_{i}\right)\right), \quad i \in \mathbb{Z}, \\
x_{t_{0}}=\xi=\{\xi(t):-\theta \leq t \leq 0\}
\end{array}\right.
$$


where $\mathbb{Z}$ is the set of integer, for any $i, k \in \mathbb{Z}$, and the sequence $\left\{t_{i}\right\}$ is such that the derived sequence $\left\{t_{i}^{k}=t_{i+k}-t_{i}\right\}$ is equipotentially almost periodic. Moreover, $A: D(A) \subset \mathbb{H} \rightarrow \mathbb{H}$ is a linear bounded operator, $\rho(A)$ is the resolvent set of $A$, and for $\lambda \epsilon$ $\rho(A), R(\lambda, A)$ is the resolvent of $A$. In addition, $b, \sigma$, and $I_{i}$ are appropriate functions, $x_{t}(\cdot):[-\theta, 0] \rightarrow \mathbb{H}$ is given by $x_{t}(s)=x(t+$ $s)$, for any $s \in[-\theta, 0]$, and $\xi \in \mathcal{C}_{\theta}$ is an $\mathcal{F}_{t_{0}}$-measurable random variable such that $E\|\xi\|^{2}<\infty$. Let $\theta>0$ be a given constant and let

$\mathcal{C}_{\theta}=\{\phi:[-\theta, 0] \times \Omega \rightarrow \mathbb{H}, \phi$ be continuous everywhere except for a finite number of points $s$ at which $\phi\left(s^{-}\right)$and $\phi\left(s^{+}\right)$exist and satisfy $\left.\phi\left(s^{-}\right)=\phi(s)\right\}$, endowed with the norm

$$
\|\phi\|_{\mathcal{C}_{\theta}}=\sup _{-\theta \leq s \leq 0}\left(E\|\phi(s)\|^{2}\right)^{\frac{1}{2}}
$$

such that $\phi(s, \cdot)$ is $\mathcal{F}_{0}$-measurable for each $s \in[-\theta, 0]$ and $\sup E\|\phi(s)\|^{2}<\infty$.

$s \in[-\theta, 0]$

There are several difficulties with our problems. First, there is the delay for the impulsive stochastic differential equations. Second, about the stochastic differential equations driven by fractional Brownian motion, the classical stochastic integral failed for lack of the martingale property. Third, there is no strong solution for stochastic partial delay differential equations driven by fractional Brownian motion. The lifting space method, mild solutions, fixed point theorem, and semigroup theory will be used to overcome these difficulties.

The paper is organized as follows. In Section 2, we introduce some notations and necessary preliminaries. Section 3 is devoted to stating the existence and uniqueness of the mild square-mean piecewise almost periodic solution to (1). In Section 4, we show the stability of the mild square-mean piecewise almost periodic solution. An example is provided to illustrate the effectiveness of the results.

\section{PRELIMINARIES}

Let $\left(\mathbb{H},\|\cdot\|_{\mathbb{H}},(\cdot, \cdot)_{\mathbb{H}}\right)$ and $\left(\mathbb{K},\|\cdot\|_{\mathbb{K}},(\cdot, \cdot)_{\mathbb{K}}\right)$ denote two real separable Hilbert spaces. We denote by $\mathbb{L}(\mathbb{H}, \mathbb{K})$ the set of all linear bounded operators from $\mathbb{H}$ into $\mathbb{K}$, equipped with the usual operator norm $\|\cdot\|$ and use $|\cdot|$ to denote the Euclidean norm of a vector. In this article, we use the symbol $\|\cdot\|$ to denote the norms of operators regardless of the spaces involved when no confusion possibly arises. Let $\left(\Omega, \mathcal{F},\left\{\mathcal{F}_{t}\right\}_{t \geq 0}, P\right)$ be a filtered complete probability space satisfying the usual condition.

\subsection{Fractional Brownian Motion}

In this subsection, we briefly introduce some useful results about $\mathrm{fBm}$ and the corresponding stochastic integral taking values in a Hilbert space. For more details, refer to $\mathrm{Hu}$ [22], Mishura [23], Nualart [24], and references therein.

A real standard fractional Brownian motion $\left\{\beta^{H}(t), t \in \mathbb{R}\right\}$ with Hurst parameter $H \in(0,1)$ is a Gaussian process with continuous sample paths such that $E\left[\beta^{H}(t)\right]=0$ and

$$
E\left[\beta^{H}(t) \beta^{H}(s)\right]=\frac{1}{2}\left(|t|^{2 H}+|s|^{2 H}-|t-s|^{2 H}\right),
$$

for all $s, t \geq 0$. It is known that $\mathrm{fBm}\left\{\beta^{H}(t), t \geq 0\right\}$ with $H>\frac{1}{2}$ admits the following Wiener integral representation:

$$
\beta^{H}(t)=\int_{0}^{t} K_{H}(t, s) d W(s)
$$

where $W$ is a standard Brownian motion and the kernel $K_{H}(t, s)$ is given by

$$
K_{H}(t, s)=c_{H} \int_{s}^{t}(u-s)^{H-\frac{3}{2}}\left(\frac{u}{s}\right)^{H-\frac{1}{2}} d u, \quad s<t,
$$

where $c_{H}>0$ is a constant satisfying $E\left(\beta_{1}^{H}\right)^{2}=1$. For any function $\sigma \in L^{2}(0, T)$, the Wiener integral of $\sigma$ with respect to $\beta^{H}$ is defined by

$$
\int_{0}^{T} \sigma(s) d \beta^{H}(s)=\int_{0}^{T} K_{H}^{*} \sigma(s) d W(s),
$$

for any $T>0$, where $K_{H}^{*} \sigma(s)=\int_{s}^{T} \frac{\partial K_{H}}{\partial r}(r, s) d r$. A $\mathbb{K}$-valued, $\mathcal{F}_{t}$-adapted $\mathrm{fBm} B^{H}$ with Hurst index $H$ can be defined by

$$
B^{H}(t)=\sum_{n=1}^{\infty} \sqrt{\lambda_{n}} e_{n} \beta_{n}^{H}(t),
$$

where $\beta_{n}^{H}, n=1,2, \cdots$ are independent $\mathrm{fBms}$ with the same Hurst parameter $H \in\left(\frac{1}{2}, 1\right),\left\{e_{n}, n \in N\right\}$, which is a complete orthonormal basis in $\mathbb{K},\left\{\lambda_{n}, n \in N\right\}$ that is a bounded sequence of non-negative real numbers satisfying $Q e_{n}=\lambda_{n} e_{n}$, and $Q$ is non-negative self-adjoint trace class operator with $\operatorname{Tr} Q=\sum_{n=1}^{\infty} \lambda_{n}<+\infty$.

Let $\mathbb{L}_{2}^{0}(\mathbb{H}, \mathbb{K})$ denote the space of all $\sigma \in \mathbb{L}(\mathbb{H}, \mathbb{K})$ such that $\sigma Q^{\frac{1}{2}}$ is a Hilbert-Schmidt operator. The norm is defined by $\|\sigma\|_{\mathbb{L}_{2}^{0}}^{2}=\sum_{n=1}^{\infty}\left\|\sqrt{\lambda_{n}} \sigma e_{n}\right\|^{2}$. Generally, $\sigma$ is called a $Q$ Hilbert-Schmidt operator from $\mathbb{H}$ to $\mathbb{K}$.

Definition 2.1. Let $\sigma:[0, T] \rightarrow L_{2}^{0}(\mathbb{H}, \mathbb{K})$ such that

$$
\sum_{n=1}^{\infty}\left\|K_{H}^{*}\left(\sigma e_{n}\right)\right\|_{\mathbb{L}_{2}^{0}}^{2}<\infty
$$

then the stochastic integral of $\sigma$ with respect to $f B m B^{H}$ is defined by

$$
\begin{gathered}
\int_{0}^{t} \sigma(s) d B^{H}(s):=\sum_{n=1}^{\infty} \int_{0}^{t} \sigma(s) Q^{\frac{1}{2}} e_{n} d \beta^{H}(s) \\
=\sum_{n=1}^{\infty} \int_{0}^{t}\left(K_{H}^{*}\left(\sigma(s) Q^{\frac{1}{2}} e_{n}\right)\right)(s) d W(s) .
\end{gathered}
$$

Remark. If $\left\{\lambda_{n}\right\}_{n \in \mathbb{N}}$ is a bounded sequence of non-negative real numbers such that the nuclear operator $Q$ satisfies $Q e_{n}=\lambda e_{n}$, assuming that there exists a positive constant $K_{\sigma}$ such that $\|\sigma\|_{\mathbb{L}_{2}^{0}}^{2} \leq K_{\sigma}$ uniformly in $[0, T]$, then it is obvious that $\sum_{n=1}^{\infty}\left\|\sigma Q^{\frac{1}{2}} e_{n}\right\|^{2}$ is uniformly convergent for $t \in[0, T]$.

\subsection{Piecewise Almost Periodic Stochastic Processes}

In this subsection, we recall some notations about the squaremean piecewise almost periodic stochastic process and introduce 
some lemmas. For further details, we refer to Takens and Teissier [25] and Liu [26].

Recall that a stochastic process $X: \mathbb{R} \rightarrow L^{2}(\Omega ; \mathbb{H})$ is said to be continuous if

$$
\lim _{t \rightarrow s} E\|X(t)-X(s)\|^{2}=0,
$$

for all $s \in \mathbb{R}$, and it is said to be bounded if there exists $N>0$, such that $E\|X(t)\|^{2} \leq N$ for all $t \in \mathbb{R}$. For convenience, we list the following concepts and notations:

- $L^{2}(\Omega, \mathbb{H})$ is Banach space when it is equipped with norm $\|\cdot\|_{L^{2}(\Omega, \mathbb{H})}$.

- Let $\mathcal{T}$ be the set consisting of all real sequences $\left\{t_{i}\right\}_{i \in \mathbb{Z}}$ such that $\alpha=\inf _{i \in \mathbb{Z}}\left(t_{i+1}-t_{i}\right)>0$, and $\lim t_{i}=\infty, \lim t_{i}=-\infty$, $x\left(t_{i}^{-}\right)$, and $x\left(t_{i}^{+}\right)$represent the left and right limits of $x(t)$ at the point $t_{i}, i \in \mathbb{Z}$, respectively.

- Let $P C\left(\mathbb{R}, L^{2}(\Omega, \mathbb{H})\right)$ be the space consisting of all stochastically bounded functions $b: \mathbb{R} \rightarrow L^{2}(\Omega, \mathbb{H})$ such that $b(\cdot)$ is stochastically continuous at $t$ for any $t \notin\left\{t_{i}\right\}_{i \in \mathbb{Z}}$, and $b\left(t_{i}\right)=b\left(t_{i}^{-}\right)$for all $i \in \mathbb{Z},\left\{t_{i}\right\}_{i \in \mathbb{Z}} \in \mathcal{T}$.

- Let $P C\left(\mathbb{R} \times \mathcal{C}_{\theta}, L^{2}(\Omega, \mathbb{H})\right)$ be the space of all piecewise stochastic process $b: \mathbb{R} \times \mathcal{C}_{\theta} \rightarrow L^{2}(\Omega, \mathbb{H})$ such that

- for any $\phi \in \mathcal{C}_{\theta}, b(\cdot, \phi)$ is stochastically continuous at point $t$ for any $t \notin\left\{t_{i}\right\}_{i \in \mathbb{Z}}$ and $b\left(t_{i}, \phi\right)=b\left(t_{i}^{-}, \phi\right)$ for all $i \in \mathbb{Z}$; and $b(t, \cdot)$ is stochastically continuous at $\phi \in \mathcal{C}_{\theta}$, for $t \in \mathbb{R}$.

- For $k<i, t-t_{k}=t-t_{i}+t_{i}-t_{k} \geq t-t_{i}+(i-k) \alpha$, if $\left\{t_{i}\right\}_{i \in \mathbb{Z}} \in \mathcal{T}$, and $t_{i}<t \leq t_{i+1}$ (see [27]).

Definition 2.2. ([28]). The family of the sequence $\left\{t_{i}^{k}=t_{i+k}-\right.$ $\left.t_{i}\right\}, i \in \mathbb{Z}, k \in \mathbb{Z}$ will be called equipotentially almost periodic if for any $\varepsilon>0$; there exists a relatively dense set $\mathbb{Q}_{\varepsilon}$ of $\mathbb{R}$ and an integer $q \in \mathbb{Z}$ such that the inequality

$$
\left|t_{i+q}-t_{i}-\tau\right|<\varepsilon
$$

holds for each $\tau \in \mathbb{Q}_{\varepsilon}$ and $i \in \mathbb{Z}$.

Definition 2.3. A function $\{b(t), t \geq 0\}$ is said to be square-mean piecewise almost periodic if the following conditions are fulfilled:

a) For any $\varepsilon>0$, there exists a positive number $\delta=\delta(\varepsilon)$ such that if the points $t^{\prime}$ and $t^{\prime \prime}$ belong to the same interval of continuity and $\left|t^{\prime}-t^{\prime \prime}\right|<\delta$, then $E\left\|b\left(t^{\prime}\right)-b\left(t^{\prime \prime}\right)\right\|^{2}<\varepsilon$.

b) For any $\varepsilon>0$, there exists $l(\varepsilon)>0$, such that every interval of length $l(\varepsilon)$ contains a number $\tau$ with the property

$$
\sup _{t \in \mathbb{R}} E\|b(t+\tau)-b(t)\|^{2}<\varepsilon
$$

which satisfies the condition $\left|t-t_{i}\right|>\varepsilon, i \in \mathbb{Z}$.

Let $\mathcal{A} \mathcal{P}_{\mathcal{T}}\left(\mathbb{R}, L^{2}(\Omega ; \mathbb{H})\right)$ denote the space of all square-mean piecewise almost periodic functions. Obviously $\mathcal{A P}_{\mathcal{T}}\left(\mathbb{R}, L^{2}(\Omega ; \mathbb{H})\right)$ endowed with the supremum norm is a Banach space. Let $\mathcal{U} P C\left(\mathbb{R} ; L^{2}(\Omega ; \mathbb{H})\right)$ be the space of all functions $b \in P C\left(\mathbb{R}, L^{2}(\Omega ; \mathbb{H})\right)$ such that $b$ satisfies the condition (a) in Definition 2.3. It is easy to check that $\mathcal{U P C}\left(\mathbb{R} ; L^{2}(\Omega ; \mathbb{H})\right)$ is a Banach space with the norm

$$
\|X\|_{\infty}=\sup _{t \in \mathbb{R}}\left(E\|X(t)\|^{2}\right)^{\frac{1}{2}},
$$

for each $X \in \mathcal{U} P C\left(\mathbb{R} ; L^{2}(\Omega ; \mathbb{H})\right)$.

Definition 2.4. (compare with [28]). A sequence $\left\{x_{i}\right\}: \mathbb{Z} \rightarrow L^{2}(\Omega, \mathbb{H})$ is called square-mean almost periodic if for any $\varepsilon>0$, there exists a natural number $N=N(\varepsilon)$ such that, for each $k \in \mathbb{Z}$, there is at least one integer $p$ in the segment $[k, k+N]$, for which inequality

$$
E\left\|x_{i+p}-x_{i}\right\|^{2}<\varepsilon,
$$

holds for all $i \in \mathbb{Z}$.

Definition 2.5. The function $b(t, \varphi) \in P C\left(\mathbb{R} \times \mathcal{C}_{\theta}, L^{2}(\Omega, \mathbb{H})\right)$ is said to be square-mean piecewise almost periodic in $t \in \mathbb{R}$ uniformly in $\varphi \in \Lambda$, where $\Lambda \subseteq \mathcal{C}_{\theta}$ is compact if for any $\varepsilon>0$, there exits $l(\varepsilon, \Lambda)>0$ such that any interval of length $l(\varepsilon, \Lambda)$ contains at least a number $\tau$ for which

$$
\sup _{t \in \mathbb{R}} E\|b(t+\tau, x)-b(t, x)\|^{2}<\varepsilon,
$$

for each $x \in \Lambda, t \in \mathbb{R}$, satisfying $\left|t-t_{i}\right|>\varepsilon$. The collection of all such processes is denoted by $\mathcal{A} \mathcal{P}_{\mathcal{T}}\left(\mathbb{R} \times \mathcal{C}_{\theta}, L^{2}(\Omega, \mathbb{H})\right)$.

Lemma 2.1. Let the function $f: \mathbb{R} \times \mathcal{C}_{\theta} \rightarrow L^{2}(\Omega ; \mathbb{H})$ be squaremean piecewise almost periodic in $t \in \mathbb{R}$ uniformly for $y \in \mathcal{C}_{\theta}$, where $\Lambda \subset \mathcal{C}_{\theta}$ is compact. Iff is a Lipschitz function in the following sense,

$$
E\|f(t, y)-f(t, \tilde{y})\|<M_{2}\left(\|y-\tilde{y}\|_{\mathcal{C}_{\theta}}\right),
$$

for all $y, \tilde{y} \in \mathcal{C}_{\theta}, t \in \mathbb{R}$, and a constant $M_{2}>0$, then for any $\phi(\cdot) \in \mathcal{A P}_{\mathcal{T}}\left(\mathbb{R}, L^{2}(\Omega ; \mathbb{H})\right), f(\cdot, \phi.) \in \mathcal{A} \mathcal{P}_{\mathcal{T}}\left(\mathbb{R}, L^{2}(\Omega ; \mathbb{H})\right)$.

Proof. Noting that $\phi(t): \mathbb{R} \rightarrow L^{2}(\Omega ; \mathbb{H})$ is square-mean almost periodic, we can conclude that $\phi_{t}=\{\phi(t+s),-\theta \leq s \leq 0, \theta>0\}$ is square-mean almost periodic by Theorem 1.2.7 of [29]. Thus, for each $\varepsilon>0$, there exists a constant $l(\varepsilon)>0$ such that every interval with the length $l(\varepsilon)$ contains a number $\tau$ satisfying

$$
E\left\|\phi_{t+\tau}-\phi_{t}\right\|_{\mathcal{C}_{\theta}}^{2} \leq \frac{\varepsilon}{4 M^{2}}, \forall t \in \mathbb{R} .
$$

Noting that $f: \mathbb{R} \times \mathcal{C}_{\theta} \rightarrow L^{2}(\Omega ; \mathbb{H})$ is square-mean piecewise almost periodic, we can see that for any $\varepsilon>0$, there exits $l(\varepsilon, \Lambda)>0$ such that each interval with length $l(\varepsilon, \Lambda)$ contains at least a number $\tau$ satisfying

$$
E\left\|f\left(t+\tau, \phi_{t}\right)-f\left(t, \phi_{t}\right)\right\|^{2} \leq \frac{\varepsilon}{4}, \forall t \in \mathbb{R},
$$

for any $x \in \Lambda\left(\subseteq \mathcal{C}_{\theta}\right), t \in \mathbb{R}$ with $\left|t-t_{i}\right|>\varepsilon$. Using the elementary inequality $|a+b|^{2} \leq 2\left(|a|^{2}+|b|^{2}\right)$ and condition (3), we have

$$
\begin{aligned}
& E\left\|f\left(t+\tau, \phi_{t+\tau}\right)-f\left(t, \phi_{t}\right)\right\|^{2} \\
& \leq 2 E\left\|f\left(t+\tau, \phi_{t+\tau}\right)-f\left(t, \phi_{t+\tau}\right)\right\|^{2}+2 E\left\|f\left(t, \phi_{t+\tau}\right)-f\left(t, \phi_{t}\right)\right\|^{2} \\
& \leq 2 E\left\|f\left(t+\tau, \phi_{t+\tau}\right)-f\left(t, \phi_{t+\tau}\right)\right\|^{2}+2 M_{2}^{2} E\left\|\phi_{t+\tau}-\phi_{t}\right\|_{\mathcal{C}_{\theta}}^{2},
\end{aligned}
$$

for all $t \in \mathbb{R}$. Combining (4) and (5), one can show that 


$$
\sup _{t \in \mathbb{R}} E\left\|f\left(t+\tau, \phi_{t+\tau}\right)-f\left(t, \phi_{t}\right)\right\|^{2} \leq 2 \cdot \frac{\varepsilon}{4}+2 M^{2} \cdot \frac{\varepsilon}{4 M^{2}} \leq \varepsilon,
$$

which implies that $f\left(t, \phi_{t}\right)$ is square-mean piecewise almost periodic.

\section{EXISTENCE OF SQUARE-MEAN PIECEWISE ALMOST PERIODIC SOLUTION}

In this section, we study the existence of the square-mean piecewise almost periodic solution to (1). We first present some assumptions as follows:

(H1) Let the bounded linear operator $A$ be an infinitesimal generator of an analytic semigroup $\{S(t), t \geq 0\}$ such that

$$
\|S(t)\| \leq M e^{-\gamma t}, \quad t \geq 0
$$

for some $\gamma>0, M>0$. Moreover, $R(\lambda, A)$ is almost periodic, where $\lambda \in \rho(A)$.

(H2) Let $b \in \mathcal{A P}_{\mathcal{T}}\left(\mathbb{R} \times \mathcal{C}_{\theta}, L^{2}(\Omega, \mathbb{H})\right)$. Moreover, there exists a positive constant $M_{b}$ such that

$$
E\|b(t, x)-b(t, \tilde{x})\|^{2} \leq M_{b}\|x-\tilde{x}\|_{\mathcal{C}_{\theta}}^{2},
$$

for any $x, \tilde{x} \in \mathcal{C}_{\theta}$.

(H3) Let $\sigma \in \mathcal{A P}_{\mathcal{T}}\left(\mathbb{R} L_{2}^{0}\left(\Omega, L^{2}(\Omega, \mathbb{H})\right)\right)$ and let $\left\{I_{i} x\left(t_{i}\right), i \in \mathbb{Z}\right\}$ be a square-mean piecewise almost periodic sequence satisfying

$$
E\left\|I_{i}(x)-I_{i}(y)\right\|^{2} \leq M_{I} E\|x-y\|^{2} .
$$

for some positive constant $M_{I}$.

Recall the notion of a mild solution for Eq. 1.

Definition 3.1. An $\mathcal{F}_{t}$-progressive process $\{x(t)\}_{t \in \mathbb{R}}$ is called a mild solution of the system (1) on $\mathbb{R}$ if it satisfies the corresponding stochastic integral equation

$$
\begin{aligned}
x(t)=S(t) x_{0} & +\int_{t_{0}}^{t} S(t-s) b\left(s, x_{s}\right) d s \\
& +\int_{t_{0}}^{t} S(t-s) \sigma(s) d B^{H}(s) \\
& +\sum_{t_{0}<t<t_{i}}^{t_{i}} S\left(t-t_{i}\right) I_{i}\left(x\left(t_{i}\right)\right),
\end{aligned}
$$

for all $t \geq t_{0}$ and for each $t_{0} \in \mathbb{R}$.

Theorem 3.1. Let $(H 1)-(H 3)$ be satisfied. Then, (1) has a unique square-mean piecewise almost periodic mild solution whenever

$$
\Theta:=\frac{M^{2} M_{b}}{\gamma^{2}}+\frac{M^{2} M_{I}}{\left(1-e^{-\gamma \alpha}\right)^{2}}<1 .
$$

Consider the following equation:

$$
\begin{aligned}
x(t)= & \int_{-\infty}^{t} S(t-s) b\left(s, x_{s}\right) d s+\int_{-\infty}^{t} S(t-s) \sigma(s) d B^{H}(s) \\
& +\sum_{t_{i}<t} S\left(t-t_{i}\right) I_{i}\left(x\left(t_{i}\right)\right),
\end{aligned}
$$

with $t \geq t_{0}$. It is easy to verify that the above equation is equivalent to (7). Define the operator $\mathcal{L}$ on $\mathcal{A P}_{\mathcal{T}}\left(\mathbb{R}, L^{2}(\Omega, \mathbb{H})\right)$ by

$$
\begin{aligned}
\mathcal{L} x(t):= & \int_{-\infty}^{t} S(t-s) b\left(s, x_{s}\right) d s \\
& \quad+\int_{-\infty}^{t} S(t-s) \sigma(s) d B^{H}(s)+\sum_{t_{i}<t} S\left(t-t_{i}\right) I_{i}\left(x\left(t_{i}\right)\right) \\
& \equiv \Phi_{1}(t)+\Phi_{2}(t)+\Phi_{3}(t),
\end{aligned}
$$

for all $t \in \mathbb{R}$. To prove the theorem, it is sufficient to show that the next statements hold:

I) $\mathcal{L} x(t)$ is square-mean piecewise almost periodic.

II) $\mathcal{L}$ admits a unique fixed point.

Proof of Statement (I)This will be done in two steps.

Step 1. We claim that $\mathcal{L} x(t) \in \mathcal{U} P C$.

Let $i \in \mathbb{Z}$. By the uniform continuity of $S(t)$, we can see that, for any $\varepsilon>0$, there exists a number $\delta>0$ between 0 and $\min \left\{\sqrt{\frac{\varepsilon}{\tilde{b}}}, \sqrt[2 H]{\frac{\varepsilon}{\tilde{\sigma}}}\right\}$ such that

$$
\left\|S\left(t^{\prime \prime}-t^{\prime}\right)-I\right\|^{2} \leq \min \left\{\frac{\gamma^{2} \varepsilon}{\tilde{b}}, \frac{\gamma^{2 H} \varepsilon}{H^{2 H} \tilde{\sigma}}, \frac{\left(1-e^{-\gamma \alpha}\right)^{2} \varepsilon}{\tilde{I}}\right\},
$$

for all $t^{\prime}, t^{\prime \prime} \in\left(t_{i}, t_{i+1}\right), t^{\prime}<t^{\prime \prime}$ as $0<t^{\prime \prime}-t^{\prime}<\delta$, where $\tilde{b}=36 M^{2}\|b\|_{\infty}^{2}, \tilde{\sigma}=36 H(2 H-1) M^{2}\|\sigma\|_{\infty}^{2}, \tilde{I}=9 M^{2}\|I\|_{\infty}^{2} . \quad$ It follows from the inequality $|a+b+c|^{3} \leq 3\left(a^{2}+b^{2}+c^{2}\right)$ that

$$
\begin{aligned}
E \| \mathcal{L} x\left(t^{\prime}\right)- & \mathcal{L} x\left(t^{\prime \prime}\right)\left\|^{2} \leq 3 E\right\| \Phi_{1}\left(t^{\prime}\right)-\Phi_{1}\left(t^{\prime \prime}\right) \|^{2} \\
& +3 E\left\|\Phi_{2}\left(t^{\prime}\right)-\Phi_{2}\left(t^{\prime \prime}\right)\right\|^{2}+3 E\left\|\Phi_{3}\left(t^{\prime}\right)-\Phi_{3}\left(t^{\prime \prime}\right)\right\|^{2},
\end{aligned}
$$

for all $t^{\prime}, t^{\prime \prime} \in\left(t_{i}, t_{i+1}\right), t^{\prime}<t^{\prime \prime}$. By the assumptions (H1), (H2), and $(H 3)$, we have that

$$
\begin{gathered}
E\left\|\int_{t^{\prime}}^{t^{\prime \prime}} S\left(t^{\prime \prime}-s\right) b\left(s, x_{s}\right) d s\right\|^{2} \leq M^{2} \int_{t^{\prime}}^{t^{\prime \prime}} e^{-\gamma\left(t^{\prime \prime}-s\right)} \\
d s \int_{t^{\prime}}^{t^{\prime \prime}} e^{-\gamma\left(t^{\prime \prime}-r\right)} E\left\|b\left(s, x_{r}\right)\right\|^{2} d r \\
\leq M^{2}\left(t^{\prime \prime}-t^{\prime}\right)^{2} \sup _{t \in \mathbb{R}} E\left\|b\left(t, x_{t}\right)\right\|^{2},
\end{gathered}
$$

and

$$
\begin{aligned}
E\left\|\int_{t^{\prime}}^{t^{\prime \prime}} S\left(t^{\prime \prime}-s\right) \sigma(s) d B^{H}(s)\right\|^{2} & \leq H(2 H-1) M^{2}\|\sigma\|_{\infty}^{2}\left(\int_{t^{\prime}}^{t^{\prime \prime}} e^{\left.-\frac{\gamma\left(t^{\prime \prime}-s\right)}{H} d s\right)^{2 H}}\right. \\
& \leq M^{2}\left(t^{\prime \prime}-t^{\prime}\right)^{2 H} \sup _{t \in \mathbb{R}} E\left\|b\left(t, x_{t}\right)\right\|^{2},
\end{aligned}
$$

for all $t^{\prime}, t^{\prime \prime} \in\left(t_{i}, t_{i+1}\right), t^{\prime}<t^{\prime \prime}$. Moreover, we also have that 


$$
\begin{aligned}
& E\left\|\int_{-\infty}^{t^{\prime}}\left[S\left(t^{\prime}-s\right)-S\left(t^{\prime \prime}-s\right)\right] b\left(s, x_{s}\right) d s\right\|^{2} \\
& \quad=E\left\|\int_{-\infty}^{t^{\prime}}\left[I-S\left(t^{\prime \prime}-t^{\prime}\right)\right] S\left(t^{\prime}-s\right) b\left(s, x_{s}\right) d s\right\|^{2} \\
& \quad \leq M^{2}\left\|I-S\left(t^{\prime \prime}-t^{\prime}\right)\right\|^{2} \int_{-\infty}^{t^{\prime}} e^{-\gamma\left(t^{\prime}-s\right)} d s \int_{-\infty}^{t^{\prime}} e^{-\gamma\left(t^{\prime}-r\right)} E\left\|b\left(s, x_{r}\right)\right\|^{2} d r \\
& \quad \leq M^{2}\left\|I-S\left(t^{\prime \prime}-t^{\prime}\right)\right\|^{2} \gamma^{-2} \sup _{t \in \mathbb{R}} E\left\|b\left(t, x_{t}\right)\right\|^{2},
\end{aligned}
$$

and

$$
\begin{aligned}
E \| \int_{-\infty}^{t^{\prime}} & {\left[S\left(t^{\prime}-s\right)-S\left(t^{\prime \prime}-s\right)\right] \sigma(s) d B^{H}(s) \|^{2} } \\
& \leq H(2 H-1) M^{2}\left\|I-S\left(t^{\prime \prime}-t^{\prime}\right)\right\|^{2} \\
& \left(\int_{-\infty}^{t^{\prime}} e^{\left.-\frac{\gamma\left(t^{\prime}-s\right)}{H}\|\sigma(s)\|_{L_{0}^{2}}^{\frac{1}{H}} d s\right)}\right. \\
& \leq H(2 H-1) M^{2}\left\|I-S\left(t^{\prime \prime}-t^{\prime}\right)\right\|^{2}\|\sigma\|_{\infty}^{2}\left(\frac{H}{\gamma}\right)^{2 H},
\end{aligned}
$$

for all $t^{\prime}, t^{\prime \prime} \in\left(t_{i}, t_{i+1}\right), t^{\prime}<t^{\prime \prime}$. Combining these with Hölder's inequality and (9), we get that

$$
E\left\|\Phi_{1}\left(t^{\prime}\right)-\Phi_{1}\left(t^{\prime \prime}\right)\right\|^{2} \leq \frac{2 M^{2}}{\gamma^{2}} \frac{\gamma^{2} \varepsilon}{\tilde{b}}\|b\|_{\infty}^{2}+2 \delta^{2} M^{2}\|b\|_{\infty}^{2} \leq \frac{\varepsilon}{9},
$$

and

$$
\begin{aligned}
& E\left\|\Phi_{2}\left(t^{\prime}\right)-\Phi_{2}\left(t^{\prime \prime}\right)\right\|^{2} \\
& \quad \leq 2 H(2 H-1) M^{2}\left(\left\|I-S\left(t^{\prime \prime}-t^{\prime}\right)\right\|^{2}\|\sigma\|_{\infty}^{2}\left(\frac{H}{\gamma}\right)^{2 H}\right. \\
& \left.+\|\sigma\|_{\infty}^{2}\left(t^{\prime \prime}-t^{\prime}\right)^{2 H}\right) \leq \frac{\varepsilon}{9},
\end{aligned}
$$

for all $t^{\prime}, t^{\prime \prime} \in\left(t_{i}, t_{i+1}\right), t^{\prime}<t^{\prime \prime}$ provided $\left|t^{\prime \prime}-t^{\prime}\right|<\delta$. Similarly, by the assumptions (H1) and (H3) and (9), one can see that

$$
\begin{aligned}
E \| \Phi_{3}\left(t^{\prime}\right)- & \Phi_{3}\left(t^{\prime \prime}\right)\left\|^{2} \leq E\right\| \sum_{t_{i}<t^{\prime}} S\left(t^{\prime}-t_{i}\right) I_{i}\left(x\left(t_{i}\right)\right) \\
& -\sum_{t_{i}<t^{\prime \prime}} S\left(t^{\prime \prime}-t_{i}\right) I_{i}\left(x\left(t_{i}\right)\right) \|^{2} \\
\leq & E\left\|\sum_{t_{i}<t^{\prime \prime}}\left[S\left(t^{\prime}-t_{i}\right) I_{i}\left(x\left(t_{i}\right)\right)-S\left(t^{\prime \prime}-t_{i}\right) I_{i}\left(x\left(t_{i}\right)\right)\right]\right\|^{2} \\
\leq & M^{2}\left\|I-S\left(t^{\prime \prime}-t^{\prime}\right)\right\|^{2}\left(\sum_{t_{i}<t^{\prime}} e^{-\gamma\left(t^{\prime}-t_{i}\right)}\right) \\
& \left(\sum_{t_{i}<t^{\prime}} e^{-\gamma\left(t^{\prime}-t_{i}\right)} E\left\|I_{i}\left(x\left(t_{i}\right)\right)\right\|^{2}\right) \\
\leq & M^{2}\left\|I-S\left(t^{\prime \prime}-t^{\prime}\right)\right\|^{2}\left(\sum_{t_{i}<t^{\prime}} e^{-\gamma\left(t^{\prime}-t_{i}\right)}\right)^{2}\|I\|_{\infty}^{2} \\
\leq & M^{2}\left\|I-S\left(t^{\prime \prime}-t^{\prime}\right)\right\|^{2}\left(\frac{1}{1-e^{-\gamma \alpha}}\right)^{2}\|I\|_{\infty}^{2} \leq \frac{\varepsilon}{9},
\end{aligned}
$$

for all $t^{\prime}, t^{\prime \prime} \in\left(t_{i}, t_{i+1}\right), t^{\prime}<t^{\prime \prime}$ provided $\left|t^{\prime \prime}-t^{\prime}\right|<\delta$. Thus, we have shown that the estimate

$$
E\left\|\mathcal{L} x\left(t^{\prime}\right)-\mathcal{L} x\left(t^{\prime \prime}\right)\right\|^{2}<\varepsilon,
$$

holds for all $t^{\prime}, t^{\prime \prime} \in\left(t_{i}, t_{i+1}\right), t^{\prime}<t^{\prime \prime}$ provided $\left|t^{\prime \prime}-t^{\prime}\right|<\delta$, which means $\mathcal{L} x(t) \in \mathcal{U} P C$.

Step 2. We prove the almost periodicity of $\mathcal{L} x(t)$.

For $\Phi_{1}(t)$, let $t_{i}<t<t_{i+1}$; by $(H 1),(H 2)$, and Hölder's inequality, we have that

$$
\begin{aligned}
& E\left\|\Phi_{1}(t+\tau)-\Phi_{1}(t)\right\|^{2} \\
&= E\left\|\int_{-\infty}^{t+\tau} S(t+\tau-s) b\left(s, x_{s}\right) d s-\int_{-\infty}^{t} S(t-s) b\left(s, x_{s}\right) d s\right\|^{2} \\
&=E\left\|\int_{-\infty}^{t} S(t-s)\left[b\left(s+\tau, x_{s+\tau}\right)-b\left(s, x_{s}\right)\right] d s\right\|^{2} \\
& \leq E\left(\int_{-\infty}^{t} M e^{-\gamma(t-s)}\left\|b\left(s+\tau, x_{s+\tau}\right)-b\left(s, x_{s}\right)\right\| d s\right)^{2} \\
& \leq \frac{M^{2}}{\gamma}\left(\sum_{j=-\infty}^{i-1} \int_{t_{j}+\eta}^{t_{j+1}-\eta} e^{-\gamma(t-s)} E\left\|b\left(s+\tau, x_{s+\tau}\right)-b\left(s, x_{s}\right)\right\|^{2} d s\right. \\
& \quad+\sum_{j=-\infty}^{i-1} \int_{t_{j}}^{t_{j}+\eta} e^{-\gamma(t-s)} E\left\|b\left(s+\tau, x_{s+\tau}\right)-b\left(s, x_{s}\right)\right\|^{2} d s \\
& \quad+\sum_{j=-\infty}^{i-1} \int_{t_{j}+1-\eta}^{t_{j+1}} e^{-\gamma(t-s)} E\left\|b\left(s+\tau, x_{s+\tau}\right)-b\left(s, x_{s}\right)\right\|^{2} d s \\
&\left.\quad+\int_{t_{i}}^{t_{i}+\eta} e^{-\gamma(t-s)} E\left\|b\left(s+\tau, x_{s+\tau}\right)-b\left(s, x_{s}\right)\right\|^{2} d s\right),
\end{aligned}
$$

where $\eta=\min \left\{\varepsilon, \frac{\alpha}{2}\right\}$. By Lemma 2.1 and $(H 2)$, we find that for any $\varepsilon>0$ and $i \in \mathbb{Z}$, there exists a real number $l(\varepsilon, \Lambda)>0$ such that every interval of length $l(\varepsilon, \Lambda)$ contains at least a constant $\tau$ and

$$
E\left\|b\left(t+\tau, x_{t+\tau}\right)-b\left(t, x_{t}\right)\right\|^{2}<\varepsilon, \quad \forall t \in \mathbb{R},
$$

for each $x \in \Lambda,\left|t-t_{i}\right|>\varepsilon$, since $b \in \mathcal{A P}_{\mathcal{T}}\left(\mathbb{R}, L^{2}(\Omega, \mathbb{H})\right)$, where $\Lambda \subset \mathcal{C}_{\theta}$ is compact.

For $s \in\left[t_{j}+\eta, t_{j+1}-\eta\right], j \in \mathbb{Z}, j \leq i, t-s \geq t-t_{i}+t_{i}-\left(t_{j+1}-\eta\right) \geq$ $t-t_{i}+\alpha(i+j-1)+\eta$, we have

$$
\begin{aligned}
& \sum_{j=-\infty}^{i-1} \int_{t_{j}+\eta}^{t_{j+1}-\eta} e^{-\gamma(t-s)} E\left\|b\left(s+\tau, x_{s+\tau}\right)-b\left(s, x_{s}\right)\right\|^{2} d s \\
& \leq \varepsilon \sum_{j=-\infty}^{i-1} \int_{t_{j}+\eta}^{t_{j+1}-\eta} e^{-\gamma(t-s)} d s \leq \frac{\varepsilon}{\gamma} \sum_{j=-\infty}^{i-1} e^{-\gamma\left(t-t_{j+1}+\eta\right)} \\
& \leq \frac{\varepsilon}{\gamma} \sum_{j=-\infty}^{i-1} e^{-\gamma \alpha(i-j+1)} \leq \frac{\varepsilon}{\gamma\left(1-e^{-\gamma \alpha}\right)} .
\end{aligned}
$$


For $s \in\left[t_{j}, t_{j}+\eta\right], j \in \mathbb{Z}, j \leq i$, by the mean value theorem of integral, we get that

$$
\begin{aligned}
\sum_{j=-\infty}^{i-1} \int_{t_{j}}^{t_{j}+\eta} e^{-\gamma(t-s)} E\left\|b\left(s+\tau, x_{s+\tau}\right)-b\left(s, x_{s}\right)\right\|^{2} d s \\
\leq 2 \sup _{s \in \mathbb{R}} E\left\|b\left(s, x_{s}\right)\right\|^{2} \sum_{j=-\infty}^{i-1} \int_{t_{j}}^{t_{j}+\eta} e^{-\gamma(t-s)} d s \leq 2\|b\|_{\infty}^{2} \varepsilon e^{\gamma \eta} \\
\quad \sum_{j=-\infty}^{i-1} e^{-\gamma\left(t-t_{j}\right)} \\
\leq 2\|b\|_{\infty}^{2} \varepsilon e^{\gamma \eta} e^{\gamma\left(t-t_{i}\right)} \sum_{j=-\infty}^{i-1} e^{-\gamma \alpha(i-j)} \leq \frac{2\|b\|_{\infty}^{2} e^{\frac{\gamma}{2}}}{1-e^{-\gamma \alpha}} \varepsilon .
\end{aligned}
$$

Similarly, we can show that

$$
\begin{array}{r}
\sum_{j=-\infty}^{i-1} \int_{t_{j}+1-\eta}^{t_{j+1}} e^{-\gamma(t-s)} E\left\|b\left(s+\tau, x_{s+\tau}\right)-b\left(s, x_{s}\right)\right\|^{2} d s \leq C_{1} \varepsilon \\
\quad \int_{t_{i}}^{t_{i}+\eta} e^{-\gamma(t-s)} E\left\|b\left(s+\tau, x_{s+\tau}\right)-b\left(s, x_{s}\right)\right\|^{2} d s \leq C_{2} \varepsilon
\end{array}
$$

where $C_{1}, C_{2}$ are two positive constants. Thus, we have introduced the next estimate:

$$
E\left\|\Phi_{1}(t+\tau)-\Phi_{1}(t)\right\|^{2}<N_{1} \varepsilon
$$

where $N_{1}$ is a positive constant, which implies that $\Phi_{1}(t)$ is square-mean piecewise almost periodic.

We now show that $\Phi_{2}(t)$ is square-mean piecewise almost periodic. Recall that $t \mapsto \sigma(t)$ is piecewise almost periodic if for each $\varepsilon>0$ there exists a real number $l(\varepsilon)>0$ such that the estimate

$$
\|\sigma(s+\tau)-\sigma(s)\|^{2}<\varepsilon, \forall t \in \mathbb{R},\left|t-t_{i}\right|>\varepsilon, i \in \mathbb{Z}
$$

holds for every interval of length $l(\varepsilon)$ containing a number $\tau$. By using $(H 1)$ and the computation of $\mathrm{fBm}$, we have

$$
\begin{aligned}
& E\left\|\Phi_{2}(t+\tau)-\Phi_{2}(t)\right\|^{2} \\
= & E\left\|\int_{-\infty}^{t+\tau} S(t+\tau-s) \sigma(s) d B^{H}(s)-\int_{-\infty}^{t} S(t-s) \sigma(s) d B^{H}(s)\right\|^{2} \\
= & E\left\|\int_{-\infty}^{t} S(t-s) \sigma(s+\tau) d B^{H}(s+\tau)-\int_{-\infty}^{t} S(t-s) \sigma(s) d B^{H}(s)\right\|^{2} \\
= & E\left\|\int_{-\infty}^{t} S(t-s)[\sigma(s+\tau)-\sigma(s)] d \tilde{B}^{H}(s)\right\|^{2} \\
= & H(2 H-1) \sum_{n=1}^{\infty} \int_{-\infty}^{t} \int_{-\infty}^{t}\left\|S(t-u)[\sigma(u+\tau)-\sigma(u)] Q^{\frac{1}{2}} e_{n}\right\| \\
& \times\left\|S(t-v)[\sigma(v+\tau)-\sigma(v)] Q^{\frac{1}{2}} e_{n}\right\| \cdot|u-v|^{2 H-2} d u d v \\
\leq & H(2 H-1) M^{2} \sum_{n=1}^{\infty} \int_{-\infty}^{t} \int_{-\infty}^{t} e^{-\gamma(t-u)}\left\|[\sigma(u+\tau)-\sigma(u)] Q^{\frac{1}{2}} e_{n}\right\| \\
& \times e^{-\gamma(t-v)}\left\|[\sigma(v+\tau)-\sigma(v)] Q^{\frac{1}{2}} e_{n}\right\| \cdot|u-v|^{2 H-2} d u d v \\
\leq & H(2 H-1) M^{2}\left(\int_{-\infty}^{t}\left(e^{-\gamma(t-s)}\|\sigma(s+\tau)-\sigma(s)\|_{L_{2}^{0}}\right)^{\frac{1}{H}} d s\right)^{2 H} .
\end{aligned}
$$

Furthermore, by Hölder's inequality, we have

$$
\begin{aligned}
& E\left\|\Phi_{2}(t+\tau)-\Phi_{2}(t)\right\|^{2} \\
\leq & H(2 H-1) M^{2}\left(\int_{-\infty}^{t} e^{\frac{-\gamma(t-s)}{2 H-1}} d s\right)^{\frac{2 H-1}{2 H}} \\
& \left(\int_{-\infty}^{t} e^{-\gamma(t-s)}\|\sigma(s+\tau)-\sigma(s)\|_{L_{2}^{0}}^{2} d s\right) \\
\leq & H(2 H-1) M^{2}\left(\frac{2 H-1}{\gamma}\right)^{\frac{2 H-1}{2 H}} \\
& \left(\sum_{j=-\infty}^{i-1} \int_{t_{j}+\eta}^{t_{j+1}-\eta} e^{-\gamma(t-s)}\|\sigma(s+\tau)-\sigma(s)\|_{L_{2}^{0}}^{2} d s\right. \\
+ & \sum_{j=-\infty}^{i-1} \int_{t_{j}}^{t_{j+\eta}} e^{-\gamma(t-s)}\|\sigma(s+\tau)-\sigma(s)\|_{L_{2}^{0}}^{2} d s \\
+ & \sum_{j=-\infty}^{i-1} \int_{t_{j}+1-\eta}^{t_{j+1}} e^{-\gamma(t-s)}\|\sigma(s+\tau)-\sigma(s)\|_{L_{2}^{0}}^{2} d s \\
+ & \left.\left.\int_{t_{i}}^{t_{i}+\eta} e^{-\gamma(t-s)}\|\sigma(s+\tau)-\sigma(s)\|_{L_{2}^{0}}^{2} d s\right)\right)
\end{aligned}
$$

where $\eta=\min \left\{\varepsilon, \frac{\alpha}{2}\right\}$. In the same way as that of handling $\Phi_{1}(t)$, one can introduce the estimate

$$
E\left\|\Phi_{2}(t+\tau)-\Phi_{2}(t)\right\|^{2}<N_{2} \varepsilon
$$

where $N_{2}$ is a positive constant, and hence $\Phi_{2}(t)$ is piecewise square-mean almost periodic.

For $\Phi_{3}(t)=\sum_{t_{i}<t} S\left(t-t_{i}\right) I_{i}\left(x\left(t_{i}\right)\right), i \in \mathbb{Z}$, let $\beta_{i}=I_{i}\left(x\left(t_{i}\right)\right)$. For $t_{i}<t \leq t_{i+1},\left|t-t_{i}\right|>\varepsilon,\left|t-t_{i+1}\right|>\varepsilon, i \in \mathbb{Z}$, by (2), one has $t_{i+q+1}>t+\tau>t_{i+q}$. From (H3), it follows that $\beta_{i}$ is a squaremean almost periodic sequence, for any $\varepsilon>0$; there exists such a natural number $N=N(\varepsilon)$ that, for an arbitrary $k \in \mathbb{Z}$, there is at least one integer $p>0$ in the segment $[k, k+N]$ such that the inequality

$$
E\left\|\beta_{i+p}-\beta_{i}\right\|^{2}<\varepsilon
$$

holds for all $i \in \mathbb{Z}$. We get

$$
\begin{aligned}
& E\left\|\Phi_{3}(t+\tau)-\Phi_{3}(t)\right\|^{2} \\
= & E\left\|\sum_{t_{i}<t+\tau} S\left(t+\tau-t_{i}\right) \beta_{i}-\sum_{t_{i}<t} S\left(t-t_{i}\right) \beta_{i}\right\|^{2} \\
\leq & E\left\|S\left(t-t_{i}\right)\left(\beta_{i+q}-\beta_{i}\right)\right\|^{2} \\
\leq & M^{2} \sum_{t_{i}<t} e^{-\gamma\left(t-t_{i}\right)} \sum_{t_{i}<t} e^{-\gamma\left(t-t_{i}\right)} E\left\|\beta_{i+q}-\beta_{i}\right\|^{2} \\
\leq & \frac{M^{2} \varepsilon}{\left(1-e^{-\gamma \alpha}\right)^{2}},
\end{aligned}
$$

which implies that $\Phi_{3}(t) \in \mathcal{A} \mathcal{P}_{\mathcal{T}}\left(\mathbb{R}, L^{2}(\Omega, \mathbb{H})\right)$. Thus, we have proved that $\mathcal{L} x(t) \in \mathcal{A} \mathcal{P}_{\mathcal{T}}\left(\mathbb{R}, L^{2}(\Omega, \mathbb{H})\right)$ and $\mathcal{L} x(t)$ is squaremean piecewise almost periodic.

Proof of Statement (II). Given $B=\left\{u \in \mathcal{A P}_{\mathcal{T}}\left(\mathbb{R}, L^{2}(\Omega, \mathbb{H})\right)\right\}$ and assuming that $x(t), y(t) \in B$ are both almost periodic solutions of (1) and $x(t) \neq y(t)$, then we have 


$$
\begin{gathered}
E\|\mathcal{L} x(t)-\mathcal{L} y(t)\|^{2} \\
=E \| \int_{-\infty}^{t} S(t-s)\left[b\left(s, x_{s}\right)-b\left(s, y_{s}\right)\right] d s \\
+\sum_{t_{i}<t} S\left(t-t_{i}\right)\left[I_{i}\left(x\left(t_{i}\right)\right)-I_{i}\left(y\left(t_{i}\right)\right)\right] \|^{2} \\
\leq 2\left(E\left\|\int_{-\infty}^{t} S(t-s)\left[b\left(s, x_{s}\right)-b\left(s, y_{s}\right)\right] d s\right\|^{2}\right. \\
\left.+E\left\|\sum_{t_{i}<t} S\left(t-t_{i}\right)\left[I_{i}\left(x\left(t_{i}\right)\right)-I_{i}\left(y\left(t_{i}\right)\right)\right]\right\|^{2}\right) \\
\equiv 2\left(A_{1}+A_{2}\right) .
\end{gathered}
$$

From $(H 1),(H 2),(H 3)$ and the Cauchy-Schwarz inequality, we have that

$$
\begin{aligned}
A_{1} & \leq E\left(\int_{-\infty}^{t} M^{2} e^{-2 \gamma(t-s)}\left\|b\left(s, x_{s}\right)-b\left(s, y_{s}\right)\right\| d s\right)^{2} \\
& \leq\left(\int_{-\infty}^{t} M^{2} e^{-\gamma(t-s)} d s\right)\left(\int_{-\infty}^{t} e^{-\gamma(t-s)} E\left\|b\left(s, x_{s}\right)-b\left(s, y_{s}\right)\right\|^{2} d s\right) \\
& \leq \frac{M^{2}}{\gamma} \int_{-\infty}^{t} e^{-\gamma(t-s)} M_{b} E\left\|x_{s}-y_{s}\right\|_{\mathcal{C}_{\theta}}^{2} d s \\
& \leq \frac{M^{2}}{\gamma^{2}} M_{b} \sup _{r \in \mathbb{R}} E\|x(r)-y(r)\|^{2}=\frac{M^{2} M_{b}}{\gamma^{2}}\|x-y\|_{\infty}^{2},
\end{aligned}
$$

and

$$
\begin{aligned}
A_{2} & \leq E\left\|\sum_{t_{i}<t} M^{2} e^{-2 \gamma\left(t-t_{i}\right)}\left[I_{i}\left(x\left(t_{i}\right)\right)-I_{i}\left(y\left(t_{i}\right)\right)\right]\right\|^{2} \\
& \leq\left(\sum_{t_{i}<t} M^{2} e^{-\gamma\left(t-t_{i}\right)}\right)\left(\sum_{t_{i}<t} e^{-\gamma\left(t-t_{i}\right)} E\left\|I_{i}\left(x\left(t_{i}\right)\right)-I_{i}\left(y\left(t_{i}\right)\right)\right\|^{2}\right) \\
& \leq \frac{M^{2}}{1-e^{-\gamma \alpha}}\left(\sum_{t_{i}<t} e^{-\gamma\left(t-t_{i}\right)} M_{I} E\left\|\left(x\left(t_{i}\right)\right)-\left(y\left(t_{i}\right)\right)\right\|^{2}\right) \\
& \leq \frac{M^{2} M_{I}}{\left(1-e^{-\gamma \alpha}\right)^{2}} \sup _{r \in \mathbb{R}} E\|x(r)-y(r)\|^{2}=\frac{M^{2} M_{I}}{\left(1-e^{-\gamma \alpha}\right)^{2}}\|x-y\|_{\infty}^{2} .
\end{aligned}
$$

It follows that

$$
E\|\mathcal{L} x(t)-\mathcal{L} y(t)\|^{2} \leq \Theta\|x-y\|_{\infty}^{2},
$$

for each $t \in \mathbb{R}$, which implies that

$$
\|\mathcal{L} x(t)-\mathcal{L} y(t)\|_{\infty} \leq \sqrt[2]{\Theta}\|x-y\|_{\infty} .
$$

This means that $\mathcal{L}$ is a contraction when (8) holds and statement (II) follows.

\section{ASYMPTOTIC STABILITY}

In this section, we are interested in the asymptotical stability of the almost periodic mild solution to (1) with $t_{0}=0$. For convenience, we rewrite the equation as follows:

$$
\left\{\begin{array}{l}
d x(t)=\left[A x(t)+b\left(t, x_{t}\right)\right] d t+\sigma(t) d B^{H}(t), \quad t \in\left[t_{0}, \infty\right), \quad t \neq \pm t_{i} \quad i \in \mathbb{Z}, \\
\triangle x\left(t_{i}\right)=x\left(t_{i}^{+}\right)-x\left(t_{i}^{-}\right)=I_{i}\left(x\left(t_{i}\right)\right), \quad i \in \mathbb{Z}, \\
x_{t_{0}}=\xi=\{\xi(t):-\theta \leq t \leq 0\} .
\end{array}\right.
$$

Lemma 4.1. ([30]). Let a nonnegative piecewise continuous function $t \mapsto v(t)$ satisfy the inequality

$$
v(t) \leq C+\int_{t_{0}}^{t} u(\sigma) v(\sigma) d \sigma+\sum_{t_{0}<\sigma_{i}<t} \alpha_{i} v\left(\sigma_{i}\right),
$$

for $t \geq t_{0}$, where $C \geq 0, u(\sigma)>0, \alpha_{i} \geq 0, i \in \mathbb{Z}$, and $\sigma_{i}, i \in \mathbb{Z}$ are the first kind discontinuity points of the function $v$. Then, the following estimate holds:

$$
v(t) \leq C \prod_{t_{0}<\sigma_{i}<t}\left(1+\alpha_{i}\right) e^{\int_{t_{0}}^{t} u(\sigma) d \sigma} .
$$

Theorem 4.1. Assume that (H1) - (H3) hold. The almost periodic solutions to (15) are asymptotically stable in the square-mean sense if

$$
\frac{1}{\alpha} \ln \left(1+\frac{3 M^{2} M_{I}}{1-e^{-\gamma \alpha}}\right)-\gamma+\frac{3 M^{2} M_{b}}{\gamma}<0,
$$

Proof. Let $x(t)$ and $x^{\star}(t)$ be two square-mean piecewise almost periodic mild solutions of (15); we then have that

$$
\begin{aligned}
E\left\|x(t)-x^{*}(t)\right\|^{2}= & E \| S(t)\left[\xi-\xi^{*}\right]+\int_{0}^{t} S(t-s)\left[b\left(s, x_{s}\right)-b\left(s, x_{s}^{*}\right)\right] d s \\
& +\sum_{0<t_{i}<t} S\left(t-t_{i}\right)\left[I_{i}\left(x\left(t_{i}\right)-I_{i}\left(x^{*}\left(t_{i}\right)\right)\right] \|^{2},\right.
\end{aligned}
$$

for all $t \geq 0$. By using Cauchy-Schwartz's inequality, Fubini's theorem, and assumptions $(H 1)-(H 3)$, we deduce that

$$
\begin{aligned}
& E\left\|x(t)-x^{*}(t)\right\|^{2} \leq 3 E\left\|S(t)\left[\xi-\xi^{*}\right]\right\|^{2}+3 E\left\|\int_{0}^{t} S(t-s)\left[b\left(s, x_{s}\right)-b\left(s, x_{s}^{*}\right)\right] d s\right\|^{2} \\
&+3 E \| \sum_{0<t_{i}<t} S\left(t-t_{i}\right)\left[I_{i}\left(x\left(t_{i}\right)\right)-I_{i}\left(x^{*}\left(t_{i}\right)\right)\|\|^{2}\right. \\
& \leq 3 M^{2} e^{-2 \gamma t}\left\|\xi-\xi^{*}\right\|^{2}+3 E\left(\int_{0}^{t}\left\|M e^{-\gamma(t-s)}\left[b\left(s, x_{s}\right)-b\left(s, x_{s}^{*}\right)\right]\right\| d s\right)^{2} \\
&+3 E \| \sum_{0<t_{i}<t} M e^{-\gamma\left(t-t_{i}\right)}\left[I_{i}\left(x\left(t_{i}\right)\right)-I_{i}\left(x^{*}\left(t_{i}\right)\right) \|^{2}\right. \\
& \leq 3 M^{2} e^{-\gamma t}\left\|\xi-\xi^{*}\right\|^{2}+3 \int_{0}^{t} M^{2} e^{-\gamma(t-s)} d s \int_{0}^{t} e^{-\gamma(t-s)} E\left\|b\left(s, x_{s}\right)-b\left(s, x_{s}^{*}\right)\right\|^{2} d s \\
&+3\left(\sum_{0<t_{i}<t} M^{2} e^{-\gamma\left(t-t_{i}\right)}\right)\left(\sum_{0<t_{i}<t} e^{-\gamma\left(t-t_{i}\right)} E\left\|I_{i}\left(x\left(t_{i}\right)\right)-I_{i}\left(x^{*}\left(t_{i}\right)\right)\right\|^{2}\right) \\
& \leq 3 M^{2} e^{-\gamma t}\left\|\xi-\xi^{*}\right\|^{2}+\frac{3 M^{2}}{\gamma} M_{b} \int_{0}^{t} e^{-\gamma(t-s)}\left\|x_{s}-x_{s}^{*}\right\|_{\mathcal{C}_{\theta}}^{2} d s \\
&+\frac{3 M^{2}}{1-e^{-\gamma \alpha}} M_{I}\left(\sum_{0<t_{i}<t} e^{-\gamma\left(t-t_{i}\right)} E\left\|x\left(t_{i}\right)-x^{*}\left(t_{i}\right)\right\|^{2}\right) \\
& \leq 3 M^{2} e^{-\gamma t}\left\|\xi-\xi^{*}\right\|^{2}+\frac{3 M^{2}}{\gamma} M_{b} \int_{0}^{t} e^{-\gamma(t-s)}\left(\sup _{0 \leq r \leq s} E\left\|x(r)-x^{*}(r)\right\|^{2}\right) d s \\
&+\frac{3 M^{2}}{1-e^{-\gamma \alpha}} M_{I}\left(\sum_{0<t_{i}<t} e^{-\gamma\left(t-t_{i}\right)} E\left\|x\left(t_{i}\right)-x^{*}\left(t_{i}\right)\right\|^{2}\right),
\end{aligned}
$$

for $t \geq 0$. Multiplying both sides of the above inequality by $e^{\gamma t}$, we get

$$
\begin{aligned}
& e^{\gamma t} E\left\|x(t)-x^{*}(t)\right\|^{2} \leq 3 M^{2}\left\|\xi-\xi^{*}\right\|^{2} \\
& +\frac{3 M^{2}}{\gamma} M_{b} \int_{0}^{t} e^{\gamma s}\left(\sup _{0 \leq r \leq s} E\left\|x(r)-x^{*}(r)\right\|^{2}\right) d s \\
& +\frac{3 M^{2}}{1-e^{-\gamma \alpha}} M_{I}\left(\sum_{0<t_{i}<t} e^{\gamma t_{i}} E\left\|x\left(t_{i}\right)-x^{*}\left(t_{i}\right)\right\|^{2}\right),
\end{aligned}
$$


for $t \geq 0$, which implies that

$$
\begin{aligned}
& \sup _{0 \leq s \leq t} e^{\gamma s} E\left\|x(s)-x^{*}(s)\right\|^{2} \leq 3 M^{2}\left\|\xi-\xi^{*}\right\|^{2}+\frac{3 M^{2}}{\gamma} M_{b} \int_{0}^{t} \\
& \quad \times \sup _{0 \leq r \leq s} e^{\gamma s} E\left\|x(r)-x^{*}(r)\right\|^{2} d s \\
& \quad+\frac{3 M^{2}}{1-e^{-\gamma \alpha}} M_{I}\left(\sum_{0<t_{i}<t} \sup _{0 \leq r \leq t_{i}} e^{\gamma r} E\left\|x(r)-x^{*}(r)\right\|^{2}\right),
\end{aligned}
$$

for $t \geq 0$. Combining this with Lemma 4.1, we get that

$$
\begin{aligned}
& \sup _{0 \leq s \leq t} e^{\gamma s} E\left\|x(s)-x^{*}(s)\right\|^{2} \\
& \leq 3 M^{2}\left\|\xi-\xi^{*}\right\|^{2} \prod_{0<t_{i}<t}\left(1+\frac{3 M^{2}}{1-e^{-\gamma \alpha}} M_{I}\right) e^{\int_{0}^{t} \frac{3 M^{2}}{\gamma} M_{b} d \sigma} \\
& \leq 3 M^{2}\left\|\xi-\xi^{*}\right\|^{2}\left(1+\frac{3 M^{2}}{1-e^{-\gamma \alpha}} M_{I}\right)^{\frac{t}{\alpha}} \frac{3 M^{2}}{\gamma} M_{b} t,
\end{aligned}
$$

for $t \geq 0$. So,

$$
e^{\gamma t} E\left\|x(t)-x^{*}(t)\right\|^{2} \leq 3 M^{2}\left\|\xi-\xi^{*}\right\|^{2}\left(1+\frac{3 M^{2}}{1-e^{-\gamma \alpha}} M_{I}\right)^{\frac{t}{\alpha}} e^{\frac{3 M^{2}}{\gamma} M_{b} t},
$$

for $t \geq 0$. Thus, we get the desired estimate

$$
\begin{gathered}
E\left\|x(t)-x^{*}(t)\right\|^{2} \leq 3 M^{2}\left\|\xi-\xi^{*}\right\|^{2}\left(1+\frac{3 M^{2}}{1-e^{-\gamma \alpha}} M_{I}\right)^{\frac{t}{\alpha}} e^{\frac{3 M^{2}}{\gamma} M_{b} t} e^{-\gamma t} \\
\leq 3 M^{2}\left\|\xi-\xi^{*}\right\|^{2} e^{\left[\frac{1}{\alpha} \ln \left(1+\frac{3 M^{2} M_{I}}{1-e^{-\gamma \alpha}}\right)-\gamma+\frac{3 M^{2} M_{b}}{\gamma}\right] t},
\end{gathered}
$$

and the square-mean piecewise almost periodic solution of (15) is asymptotically stable in the square-mean sense because of (16). This completes the proof.

\section{AN EXAMPLE}

Consider the semilinear impulsive stochastic partial functional differential equations of the following form:

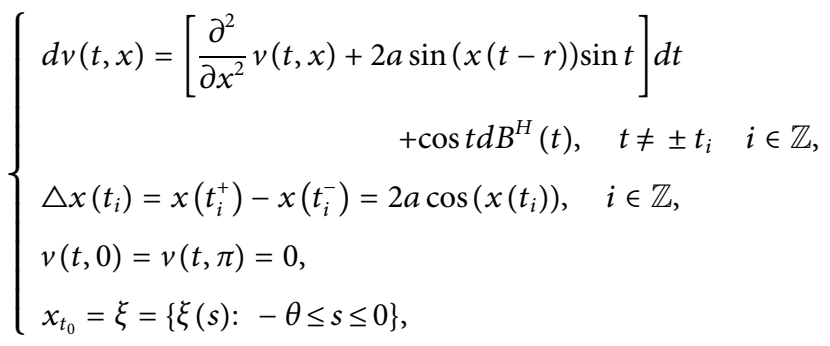

where $r$ is a constant and $B^{H}(t)$ is a fractional Brownian motion. Denote $X=L^{2}\left(\Omega, L^{2}([0, \pi])\right)$ and define $A: D(A) \subseteq X \rightarrow X$ given by $A=\frac{\partial^{2}}{\partial x^{2}}$ with the following domain:

$$
\begin{gathered}
D(A)=\left\{v(\cdot) \in X: v^{\prime \prime} \in X, v^{\prime} \in X\right. \text { are absolutely continous on } \\
[0, \pi]\} .
\end{gathered}
$$

It is well known that a strongly continuous semigroup $\{S(t)\}_{t \geq 0}$ generated by the operator $A$ satisfies $\|S(t)\| \leq e^{-t}$, for $t \geq 0$. Take

$$
b\left(t, x_{t}\right)=2 a \sin \left(x_{t}\right) \sin t,
$$

and

$$
I_{i}\left(x\left(t_{i}\right)\right)=2 a \cos \left(x\left(t_{i}\right)\right) .
$$

Thus, one has

$$
E\left\|b\left(t, x_{t}\right)-b\left(t, y_{t}\right)\right\|^{2} \leq 4 a^{2}\left\|x_{t}-y_{t}\right\|_{\mathcal{C}_{\theta}}^{2},
$$

and

$$
\left\|I_{i}(x)-I_{i}(y)\right\|^{2} \leq 4 a^{2}\|x-y\|^{2} .
$$

Let $\alpha=1$. Then, (17) has a square-mean piecewise almost periodic mild solution, provided that $0<a^{2}<\frac{1}{16}$ by Theorem 3.1, and moreover the solution of (17) is asymptotically stable in the square-mean sense provided that $0<a^{2}<\frac{1}{36}$ by Theorem 4.1 .

\section{CONCLUSION}

In this article, we have investigated the existence and asymptotic stability of square-mean piecewise almost periodic mild solutions for a class of impulsive stochastic delay differential equations driven by fractional Brownian motion with the Hurst parameter $H \in\left(\frac{1}{2}, 1\right)$ in a Hilbert space. An example is presented to illustrate our theoretical results. Fractional Brownian motion $B^{H}$ with $H \in\left(0, \frac{1}{2}\right)$ admits different Wiener integral representation from fractional Brownian motion with $H \in\left(\frac{1}{2}, 1\right)$. It is difficult to get the square-mean piecewise almost periodic mild solutions of ISDEs driven by fractional Brownian motion with $H \in\left(0, \frac{1}{2}\right)$ in a Hilbert space properly due to estimation without moment.

\section{DATA AVAILABILITY STATEMENT}

The raw data supporting the conclusion of this article will be made available by the authors, without undue reservation.

\section{AUTHOR CONTRIBUTIONS}

LG and XS carried out the mathematical studies, participated in the sequence alignment, drafted the manuscript and participated in the design of the study and performed proof of results. All authors read and approved the submitted version.

\section{FUNDING}

This work was supported by the National Natural Science Foundation of China, No. 11971101; Natural Science Foundation of Anhui Province, No.1808085MA02; and Natural Science Foundation of Bengbu University, Nos. 2020ZR04zd and BBXY2020KYQD05.

\section{ACKNOWLEDGMENTS}

The authors are indebted to Professor Litan Yan for his encouragement and helpful discussion. The authors are grateful to the referees and the associate editor for valuable comments and suggestions to improve this article. 


\section{REFERENCES}

1. Tsokos CP, Padgett WJ. Random Integral Equations with Applications to Life Sciences and Engineering. New York: Academic Press (1974).

2. Arthi G, Park JH, Jung HY. Existence and Exponential Stability for Neutral Stochastic Integrodifferential Equations with Impulses Driven by a Fractional Brownian Motion. Commun Nonlinear Sci Numer Simulation (2016) 32: 145-57. doi:10.1016/j.cnsns.2015.08.014

3. Caraballo T. Existence of Mild Solutions to Stochastic Delay Evolution Equations with a Fractional Brownian Motion and Impulses. Stoch Anal Appl (2015) 33:244-58. doi:10.1080/07362994.2014.981641

4. Cheng L, Hu L, Ren Y. Perturbed Impulsive Neutral Stochastic Functional Differential Equations. Qual Theor Dyn. Syst. (2021) 20:27. doi:10.1007/s12346-021-00469-7

5. Shi J, Li C, Sun J. Stability of Impulsive Stochastic Differential Delay Systems and its Application to Impulsive Stochastic Neural Networks. Nonlinear Anal (2011) 74:3099-111. doi:10.1016/j.na.2011.01.026

6. Liu X, Liu J, Xie W. Existence and Uniqueness Results for Impulsive Hybrid Stochastic Delay Systems. Appl Nonlinear Anal (2010) 17:37-53. doi:10.1016/ j.nahs.2009.11.004

7. Sakthivel R, Luo J. Asymptotic Stability of Nonlinear Impulsive Stochastic Differential Equations. Stat Probab Lett (2009) 79:1219-23. doi:10.1016/ j.spl.2009.01.011

8. Wu X, Yan L, Zhang W, Chen L. Exponential Stability of Impulsive Stochastic Delay Differential Systems. Discrete Dyn Nat Soc (2012) 2012:1-15. doi:10.1155/2012/296136

9. Chen H, Xiao S. Existence and Exponential Stability for Impulsive Stochastic Partial Functional Differential Equations. J Math Phys (2017) 58:032701. doi:10.1063/1.4976727

10. Ma S, Kang Y. Periodic Averaging Method for Impulsive Stochastic Differential Equations with Lévy Noise. Appl Maths Lett (2019) 93:91-7. doi:10.1016/j.aml.2019.01.040

11. Guo Y, Zhu Q, Wang F. Stability Analysis of Impulsive Stochastic Functional Differential Equations. Commun Nonlinear Sci Numer Simulation (2020) 82: 105013. doi:10.1016/j.cnsns.2019.105013

12. Li M. Multi-fractional Generalized Cauchy Process and its Application to Teletraffic. Physica A: Stat Mech its Appl (2020) 550:123982. doi:10.1016/ j.physa.2019.123982

13. Li M. Generalized Fractional Gaussian Noise and its Application to Traffic Modeling. Physica A (2020) 579:12638. doi:10.1016/j.physa.2021.126138

14. Li M. Modified Multifractional Gaussian Noise and its Application. Phys Scr (2021) 96:125002. doi:10.1088/1402-4896/ac1cf6

15. Zachevsky I, Zeevi YY. Single-Image Superresolution of Natural Stochastic Textures Based on Fractional Brownian Motion. IEEE Trans Image Process (2014) 23:2096-108. doi:10.1109/TIP.2014.2312284

16. Ksendal B. Fractional Brownian Motion in Finance. London: Springer (2008).

17. Heydari MH, Mahmoudi MR, Shakiba A, Avazzadeh Z. Chebyshev Cardinal Wavelets and Their Application in Solving Nonlinear Stochastic Differential Equations with Fractional Brownian Motion. Commun Nonlinear Sci Numer Simulation (2018) 64:98-121. doi:10.1016/j.cnsns.2018.04.018
18. Heydari MH, Avazzadeh Z, Mahmoudi MR. Chebyshev Cardinal Wavelets for Nonlinear Stochastic Differential Equations Driven with Variable-Order Fractional Brownian Motion. Chaos, Solitons \& Fractals (2019) 124:105-24. doi:10.1016/j.chaos.2019.04.040

19. Li Z, Xu L, Zhou J. Viability for Coupled SDEs Driven by Fractional Brownian Motion. Appl Maths Optimization (2021) 84:55C98. doi:10.1007/s00245-02109761-z

20. Cui J, Yan L. Controllability of Neutral Stochastic Evolution Equations Driven by Fractional Brownian Motion. Acta Mathematica Scientia (2017) 37:108-18 doi:10.1016/s0252-9602(16)30119-9

21. Biagini F, Hu Y, Øksendal B, Zhang T. Stochastic Calculus for Fractional Brownian Motion and Applications. New York: Springer (2008).

22. $\mathrm{Hu} \mathrm{Y}$. Integral Transformations and Anticipative Calculus for Fractional Brownian Motions. Mem AMS (2005) 175:825. doi:10.1090/memo/0825

23. Mishura Y. Stochastic Calculus for Fractional Brownian Motion and Related. New York: Springer (2008).

24. Nurlart D. The Malliavin Calculus and Related Topics. New York: Springer (2006).

25. Stamov GT. Almost Periodic Solutions of Impulsive Differential Equations. New York: Springer (2012).

26. Liu J, Zhang C. Existence and Stability of Almost Periodic Solutions to Impulsive Stochastic Differential Equations. CUBO (2013) 15:77-96. doi:10.4067/s0719-06462013000100005

27. Henríquez HR, De Andrade B, Rabelo M. Existence of Almost Periodic Solutions for a Class of Abstract Impulsive Differential Equations. ISRN Math Anal (2011) 2011:1-21. doi:10.5402/2011/632687

28. Samoilenko AM, Perestyuk NA. Impulsive Differential Equations. Singapore: World Scientific (1995).

29. Yoshizawa T. Stability Theory and Existence of Periodic Sotlutions and Almost Periodic Solutions. New York: Springer (1975).

30. Wang Y, Liu Z. Almost Periodic Solutions for Stochastic Differential Equations with Lévy Noise. Nonlinearity (2012) 25:2803-21. doi:10.1088/0951-7715/25/ $10 / 2803$

Conflict of Interest: The authors declare that the research was conducted in the absence of any commercial or financial relationships that could be construed as a potential conflict of interest.

Publisher's Note: All claims expressed in this article are solely those of the authors and do not necessarily represent those of their affiliated organizations, or those of the publisher, the editors and the reviewers. Any product that may be evaluated in this article, or claim that may be made by its manufacturer, is not guaranteed or endorsed by the publisher.

Copyright (C) 2021 Gao and Sun. This is an open-access article distributed under the terms of the Creative Commons Attribution License (CC BY). The use, distribution or reproduction in other forums is permitted, provided the original author(s) and the copyright owner(s) are credited and that the original publication in this journal is cited, in accordance with accepted academic practice. No use, distribution or reproduction is permitted which does not comply with these terms. 